\title{
Long-term outcomes of pediatric arteriovenous malformations: the 30-year Pittsburgh experience
}

\author{
Michael M. McDowell, MD, ${ }^{1,2}$ Nitin Agarwal, MD, ${ }^{2}$ Gordon Mao, MD, ${ }^{3}$ Stephen Johnson, MD, ${ }^{2}$ \\ Hideyuki Kano, MD, PhD, ${ }^{2}$ L. Dade Lunsford, MD, ${ }^{2}$ and Stephanie Greene, MD ${ }^{1,2}$ \\ 'Division of Neurological Surgery, Children's Hospital of Pittsburgh; ${ }^{2}$ Department of Neurological Surgery, University of Pittsburgh \\ Medical Center; and ${ }^{3}$ Department of Neurological Surgery, Allegheny Health Network, Pittsburgh, Pennsylvania
}

\begin{abstract}
OBJECTIVE The study of pediatric arteriovenous malformations (pAVMs) is complicated by the rarity of the entity. Treatment choice has often been affected by the availability of different modalities and the experience of the providers present. The University of Pittsburgh experience of multimodality treatment of pAVMs is presented.
\end{abstract}

METHODS The authors conducted a retrospective cohort study examining 212 patients with pAVM presenting to the University of Pittsburgh between 1988 and 2018, during which patients had access to surgical, endovascular, and radiosurgical options. Univariate analysis was performed comparing good and poor outcomes. A poor outcome was defined as a modified Rankin Scale ( $m R S)$ score of $\geq 3$. Multivariate analysis via logistic regression was performed on appropriate variables with a $p$ value of $\leq 0.2$. Seventy-five percent of the cohort had at least 3 years of follow-up.

RESULTS Five patients (2.4\%) did not receive any intervention, 131 (61.8\%) had GKRS alone, 14 (6.6\%) had craniotomies alone, and $2(0.9 \%)$ had embolization alone. Twenty-two (10.4\%) had embolization and Gamma Knife radiosurgery (GKRS); 20 (9.4\%) had craniotomies and GKRS; 8 (3.8\%) had embolization and craniotomies; and 10 (4.7\%) had embolization, craniotomies, and GKRS. Thirty-one patients (14.6\%) were found to have poor outcome on follow-up. The multivariate analysis performed in patients with poor outcomes was notable for associations with no treatment (OR 18.9, $p=0.02)$, hemorrhage requiring craniotomy for decompression alone (OR $6, p=0.03)$, preoperative mRS score (OR 2.1, $p=0.004)$, and Spetzler-Martin score (OR 1.8, $p=0.0005)$. The mean follow-up was $79.7 \pm 62.1$ months. The confirmed radiographic obliteration rate was $79.4 \%$ and there were 5 recurrences found on average 9.5 years after treatment.

CONCLUSIONS High rates of long-term functional independence (mRS score of $\leq 2$ ) can be achieved with comprehensive multimodality treatment of PAVMs. At this center there was no difference in outcome based on treatment choice when accounting for factors such as Spetzler-Martin grade and presenting morbidity. Recurrences are rare but frequently occur years after treatment, emphasizing the need for long-term screening after obliteration.

https://thejns.org/doi/abs/10.3171/2020.3.PEDS19614

KEYWORDS arteriovenous malformations; pediatric; outcomes; craniotomy; hemorrhage; Gamma Knife radiosurgery; embolization; vascular disorders

$\mathrm{P}$ EDIATRIC arteriovenous malformations (pAVMs) are a challenging entity in which hemorrhagic presentation occurs in more than half of cases. ${ }^{1-7}$ There is little consensus regarding the management of pAVMs beyond initial stabilization following rupture..$^{2-6,8}$ Observation, surgical removal, stereotactic radiosurgery, embolization, or a combination of these options have often been chosen at least partially based on the availability of interventions and the preferences of the practitioners involved. The availability of Gamma Knife radiosurgery (GKRS) as an adjunct or alternative to resection or embolization has had substantial effects on the management of pAVMs, particularly in cases in which the potential for operative morbidity is excessive. Our center has a more than 3-decade history of multimodality management of AVMs in which all of the current management options have been used. ${ }^{9}$ We sought to characterize the long-term outcomes of our cohort in the multimodality era.

\section{Methods}

We conducted a retrospective cohort study of pAVM

ABBREVIATIONS GKRS = Gamma Knife radiosurgery; $\mathrm{mRS}=$ modified Rankin Scale; $\mathrm{pAVM}=$ pediatric arteriovenous malformation.

SUBMITTED October 22, 2019. ACCEPTED March 6, 2020.

INCLUDE WHEN CITING Published online May 15, 2020; DOI: 10.3171/2020.3.PEDS19614. 
outcomes in patients cared for at the University of Pittsburgh and Children's Hospital of Pittsburgh during a 30-year interval (January 1, 1988-December 31, 2018). Retrospective review of clinical data was approved under the University of Pittsburgh Medical Center Institutional Review Board guidelines. The presenting symptoms, long-term modified Rankin Scale (mRS) outcome score, patient demographic information, management strategies, angioarchitecture and other radiographic characteristics, and long-term outcomes were reviewed. Recurrences and post-treatment hemorrhages were documented. ${ }^{10}$

\section{Definitions}

Pediatric patients were defined as individuals presenting with a pAVM between the ages of 0 and 18 years. Patients with other cerebrovascular lesions including vein of Galen malformations, dural arteriovenous fistulas, cavernous malformations, venous angiomas, and sinus pericranii were excluded from this study. A total of 212 patients met inclusion criteria. A poor outcome was defined as an $\mathrm{mRS}$ score of $\geq 3$, representing the absence of functional independence..$^{10}$ Obliteration was defined as absence of evidence of patent angioarchitecture on CTA, MRA, or formal diagnostic cerebral angiogram at least 6 months after treatment. Obliteration was secondarily examined, including only patients with radiographic follow-up of at least 3 years. All pAVM recurrences were confirmed by formal diagnostic cerebral angiogram. All recurrences had imaging at least 6 months post-treatment documenting obliteration of the prior pAVM, including at least one formal angiogram documenting obliteration. Aneurysms were defined as feeding artery, nonfeeding artery, venous, or intranidal.

\section{Statistical Analysis}

Univariate analysis was performed comparing good and poor outcomes via Mann-Whitney, Kruskal-Wallis, chi-square, and Fisher's exact test as appropriate. Multivariate analysis via logistic regression was performed on appropriate variables with a $\mathrm{p}$ value of $\leq 0.2$. All statistical analysis in the text and tables refers to comparisons between good and poor outcome groups for designated variables unless specifically stated otherwise. Statistical tests were performed using the Statistical Analysis System (SAS, version 9.4).

\section{Results}

Of the 212 patients, $31(14.6 \%)$ had an mRS score of $\geq 3$ on most recent follow-up, and 181 (85.4\%) had an $\mathrm{mRS}$ score of $\leq 2$ on most recent follow-up. One hundred nine patients (51.4\%) were male and $103(48.6 \%)$ were female, with 17 male and 14 female patients having poor outcomes $(p=0.68)$. The mean age on presentation was 11.3 years, with the mean age of patients with good outcome being 11.6 years versus 9.6 years in patients with poor outcome $(\mathrm{p}=0.015)$. The median $\mathrm{mRS}$ score on presentation for the entire cohort was 1 , with a median presenting mRS score of 1 for patients with good long-term outcomes, and a median presenting mRS score of 3 for patients with poor long-term outcomes $(\mathrm{p}<0.0001)$ (Table
TABLE 1. Baseline demographic and presenting information in 212 patients with pAVMs grouped by long-term $\mathrm{mRS}$ score

\begin{tabular}{lcccc}
\hline \multicolumn{1}{c}{ Characteristic } & Total & mRS 0-2 & mRS 3-6 & p Value \\
\hline No. of patients & 212 & 181 & 31 & \\
\hline Male & 109 & 92 & 17 & 0.68 \\
\hline Female & 103 & 89 & 14 & \\
\hline Mean age (yrs) & $11.3 \pm 4.3$ & $11.6 \pm 4.1$ & $9.6 \pm 5.90$ & 0.015 \\
\hline Median presenting & 1 & 1 & 3 & $<0.0001$ \\
mRS score & & & & \\
\hline 0 & 16 & 14 & 2 & \\
\hline 1 & 110 & 106 & 4 & \\
\hline 2 & 21 & 21 & 0 & \\
\hline 3 & 32 & 21 & 11 & \\
\hline 4 & 12 & 6 & 6 & \\
\hline 5 & 21 & 13 & 8 & \\
\hline
\end{tabular}

Age is expressed as the mean $\pm \mathrm{SD}$. Boldface type indicates statistical significance.

1). Seventeen patients (8\%) were asymptomatic-their lesions were discovered during imaging for other diagnoses-and 195 (92\%) were symptomatic. Overall, patients with motor deficits and obtundation were more likely to have a poor outcome, whereas asymptomatic patients and patients presenting with seizures, headaches, altered mental status, and visual field cuts were more likely to have a good long-term outcome ( $p<0.0001)$. One hundred twenty-eight patients $(60.4 \%)$ had pAVM-associated hemorrhage on presentation, with $102(56.4 \%)$ of 181 patients in the good outcome group and $26(83.9 \%)$ of 31 patients in the poor outcome group presenting with hemorrhage $(\mathrm{p}$ $=0.01$ ). The 5 patients without hemorrhage who had poor outcome included 1 patient who developed progressive epilepsy and hemiparesis from pAVM mass effect, 1 patient whose family refused treatment on incidental presentation and who suffered a fatal hemorrhage subsequently, 1 patient who had persistent deficits from brainstem mass effect even with GKRS, and 2 patients who suffered hemorrhage years after treatment. Fifty (73.5\%) of 68 patients less than 10 years of age had a hemorrhagic presentation $(\mathrm{p}=0.0067)($ Table 2$)$. The mean follow-up was $79.7 \pm$ 62.1 months. Only $13(6.1 \%)$ patients had less than 1 year of clinical follow-up, of whom 2 died.

\section{Radiological Features}

One hundred patients (47.2\%) had left-sided lesions, 91 (42.9\%) had right-sided lesions, and 20 (9.4\%) lesions were midline. One hundred eighty-four patients $(86.8 \%)$ had supratentorial lesions and 28 (13.2\%) had infratentorial lesions; of the infratentorial lesions 9 (29\%) had poor outcomes and $22(71 \%)$ had good outcomes, compared to 162 good outcomes $(88 \%)$ and 22 poor outcomes $(12 \%)$ in supratentorial lesions $(\mathrm{p}=0.005)$. One hundred thirty-four patients had superficial lesions and 78 patients had deep lesions; of the patients with deep lesions, 19 (24\%) had poor outcomes and 59 (76\%) had good outcomes compared to 122 good outcomes (91\%) and 12 poor outcomes 
TABLE 2. Presenting symptoms, frequency of hemorrhage, and frequency of emergency CSF diversion in patients with pAVMs

\begin{tabular}{lrrrc}
\hline \multicolumn{1}{c}{ Presentation } & Total & $\mathrm{mRS} 0-2$ & $\mathrm{mRS} 3-6$ & $\mathrm{p} \mathrm{Value}$ \\
\hline $\begin{array}{l}\text { All primary presenting } \\
\text { symptoms }\end{array}$ & & & & $<0.0001$ \\
\hline$\quad$ Altered mentation & 9 & 9 & 0 & 0.36 \\
\hline Aphasia & 3 & 2 & 1 & 0.38 \\
\hline Ataxia & 5 & 3 & 2 & 0.16 \\
\hline Cranial nerve palsy & 9 & 8 & 1 & 0.99 \\
\hline Visual field cut & 9 & 9 & 0 & 0.36 \\
\hline Headache & 62 & 60 & 2 & $\mathbf{0 . 0 0 2}$ \\
\hline$\quad$ Motor deficit & 48 & 30 & 18 & $<0.0001$ \\
\hline$\quad$ Incidental & 17 & 15 & 2 & 0.99 \\
\hline$\quad$ Obtundation & 18 & 13 & 5 & 0.15 \\
\hline$\quad$ Seizure & 32 & 32 & 0 & 0.006 \\
\hline Hemorrhagic presentation & 128 & 102 & 26 & 0.01 \\
\hline $\begin{array}{l}\text { Hydrocephalus requiring } \\
\text { ventriculostomy on initial } \\
\text { presentation }\end{array}$ & 32 & 26 & 6 & 0.47 \\
\hline
\end{tabular}

Boldface type indicates statistical significance.

$(9 \%)$ in patients with superficial lesions $(\mathrm{p}=0.002)$. Patients with basal ganglia and brainstem lesions trended toward having a higher frequency of poor outcomes $(\mathrm{p}=$ 0.051 ). A complete description of pAVM location is provided in Table 3. In terms of venous drainage, 167 patients $(78.8 \%)$ had 1 draining vein, 32 patients $(15.1 \%)$ had 2 draining veins, and 13 patients $(6.1 \%)$ had 3 draining veins. One hundred forty-two patients $(67 \%)$ had deep venous drainage. One hundred sixteen patients (64.1\%) with good outcomes had deep venous drainage, and 26 patients $(83.9 \%)$ with poor outcomes had deep venous drainage $(\mathrm{p}=0.008)$. Thirty-eight patients $(17.9 \%)$ presented with aneurysms, of which 16 (42.1\%) were feeding artery aneurysms, $1(2.6 \%)$ was an aneurysm of an unrelated artery, and $21(55.3 \%)$ were intranidal or venous. Four patients (1.9\%) had multiple distinct pAVMs, 1 of whom had a poor outcome and 3 of whom had a good outcome ( $\mathrm{p}=$ 0.47 ). The median Spetzler-Martin grade was III, with a median score of 3 in patients with good outcomes and 4 in patients with poor outcomes $(\mathrm{p}=0.0003)$. Quantitative data regarding Spetzler-Martin grades for each group are provided in Table 3.

\section{Treatment Characteristics}

Of the 212 patients, $5(2.4 \%)$ did not receive any intervention due to family refusal, $130(61.8 \%)$ had GKRS alone, $14(6.6 \%)$ had craniotomies alone, and $1(0.9 \%)$ had embolization alone. Twenty-two (10.4\%) had embolization and GKRS, 21 (9.4\%) had craniotomies and GKRS, 9 (3.8\%) had embolization and craniotomies, and $10(4.7 \%)$ had embolization, craniotomies, and GKRS (Table 4). Of those receiving GKRS, 159 patients underwent a single GKRS treatment, 19 had 2 treatments, 4 had 3 treatments, and 1 had 4 treatments. The median marginal dose given
TABLE 3. Anatomical and angioarchitectural features in patients presenting with pAVMs

\begin{tabular}{|c|c|c|c|c|}
\hline Feature & Total & mRS 0-2 & mRS 3-6 & $p$ Value \\
\hline All sidedness & & & & 0.15 \\
\hline $\mathrm{Lt}$ & 100 & 82 & 18 & 0.24 \\
\hline $\mathrm{Rt}$ & 91 & 83 & 8 & 0.049 \\
\hline Midline & 20 & 15 & 5 & 0.18 \\
\hline Bilat & 1 & 1 & 0 & 0.99 \\
\hline All locations & & & & 0.051 \\
\hline Frontal & 28 & 28 & 0 & 0.018 \\
\hline Parietal & 31 & 27 & 4 & 0.99 \\
\hline Temporal & 24 & 22 & 2 & 0.54 \\
\hline Occipital & 20 & 20 & 0 & 0.05 \\
\hline Brainstem & 17 & 11 & 6 & 0.023 \\
\hline Basal ganglia & 19 & 12 & 7 & 0.01 \\
\hline Thalamic & 35 & 30 & 5 & 0.99 \\
\hline Cerebellar & 11 & 8 & 3 & 0.21 \\
\hline Corpus callosum & 5 & 4 & 1 & 0.55 \\
\hline Intraventricular & 1 & 1 & 0 & 0.99 \\
\hline Hypothalamus & 1 & 1 & 0 & 0.99 \\
\hline Frontoparietal & 6 & 4 & 2 & 0.21 \\
\hline Frontotemporal & 2 & 2 & 0 & 0.99 \\
\hline Parieto-occipital & 5 & 5 & 0 & 0.99 \\
\hline Temporo-occipital & 4 & 4 & 0 & 0.99 \\
\hline Temporoparietal & 3 & 2 & 1 & 0.38 \\
\hline No. of draining veins & & & & 0.1 \\
\hline 1 & 167 & 142 & 25 & \\
\hline 2 & 32 & 30 & 2 & \\
\hline 3 & 13 & 9 & 4 & \\
\hline Deep drainage & 142 & 116 & 26 & 0.008 \\
\hline Multiple AVMs & 4 & 3 & 1 & 0.47 \\
\hline Aneurysm & 38 & 34 & 4 & 0.4 \\
\hline Feeding artery & 16 & 14 & 2 & 0.99 \\
\hline Unrelated artery & 1 & 1 & 0 & 0.99 \\
\hline Intranidal/postnidal venous & 21 & 19 & 2 & 0.75 \\
\hline Median Spetzler-Martin grade & 3 & 3 & 4 & 0.0003 \\
\hline I & 14 & 11 & 3 & 0.44 \\
\hline II & 54 & 48 & 6 & 0.51 \\
\hline III & 87 & 81 & 6 & 0.01 \\
\hline IV & 27 & 23 & 4 & 0.99 \\
\hline V & 30 & 18 & 12 & 0.0002 \\
\hline
\end{tabular}

Boldface type indicates statistical significance.

for initial treatment was $20 \mathrm{~Gy}$. Of the patients receiving embolization, 26 patients had a single embolization, 12 had 2 embolizations, and 4 had 3 embolizations. The date of embolization ranged from 1988 to 2015 . The principal agent for embolization was Onyx in 15 cases (36\%), coils in 8 cases (19\%), $N$-butyl cyanoacrylate in 7 cases (17\%), polyvinyl alcohol in 3 cases (7\%), and acrylic glue in 1 case $(2 \%)$. Five patients $(12 \%)$ were treated with a liquid 
TABLE 4. Treatment characteristics in patients presenting with pAVMs

\begin{tabular}{lrrrl}
\hline \multicolumn{1}{c}{ Characteristic } & Total & mRS 0-2 & mRS 3-6 & p Value \\
\hline Treatment & & & & 0.012 \\
\hline $\begin{array}{l}\text { Craniotomy, GKRS, \& } \\
\text { embolization }\end{array}$ & 10 & 6 & 4 & $\mathbf{0 . 0 4}$ \\
\hline Craniotomy only & 14 & 10 & 4 & 0.13 \\
\hline Craniotomy \& embolization & 9 & 9 & 0 & 0.61 \\
\hline Craniotomy \& GKRS & 21 & 15 & 6 & $\mathbf{0 . 0 4 1}$ \\
\hline Embolization only & 1 & 1 & 0 & 0.99 \\
\hline Embolization \& GKRS & 22 & 19 & 3 & 0.99 \\
\hline GKRS only & 130 & 118 & 12 & $\mathbf{0 . 0 0 4}$ \\
\hline No treatment & 5 & 3 & 2 & $\mathbf{0 . 0 2 5}$ \\
\hline Botface typendicates & & & &
\end{tabular}

Boldface type indicates statistical significance.

embolic agent of unknown composition and in 3 cases (7\%) the type of embolic material was not documented.

Thirty-two patients (15.1\%) required ventriculostomies at the time of presentation. Of the patients with combined craniotomies and GKRS, 10 patients who underwent craniotomy for hemorrhage and resection of AVM had persistent residual lesions requiring GKRS -5 required GKRS after residual following craniotomy, 4 required craniotomies due to persistent AVM despite GKRS, and 1 required craniotomy after rehemorrhage despite GKRS.

\section{Radiographic Outcomes}

A total of $165(79.7 \%)$ of the 207 patients who underwent some form of treatment had appropriate follow-up imaging at least 6 months after treatment. Of these 165 patients, 131 (79.4\%) had documented obliteration. Ninetyfour $(57 \%)$ had at least one formal diagnostic angiogram during follow-up documenting obliteration. One hundred thirty-five patients $(65.2 \%)$ had at least 3 years of followup imaging. Of these, $104(77 \%)$ had documented obliteration. A total of 5 recurrences (3\%) occurred among the 165 patients with documented obliterations. Two patients underwent craniotomies alone, 2 patients underwent embolization and craniotomy, and 1 patient underwent GKRS alone for the recurrences. The mean age at recurrence was 17 years (ages 4, 10, 20, 25, and 26 years), and the mean time to recurrence was 9.5 years. Four of the 5 patients with recurrences presented with hemorrhage. There was no specific location associated with recurrences, with 1 recurrence each found in the cerebellum, frontal lobe, parietal lobe, corpus callosum, and lateral ventricle. Patients who underwent craniotomy as part of their treatment had a significantly higher rate of recurrence than those undergoing GKRS alone ( $\mathrm{p}=0.0015)$.

\section{Clinical Outcomes}

On the most recent follow-up, 99 patients $(46.7 \%)$ had an mRS of $0 ; 56(26.4 \%)$ had an mRS of $1 ; 26(12.3 \%)$ had an mRS of $2 ; 22(10.4 \%)$ had an mRS of $3 ; 1(0.5 \%)$ had an mRS of 4; and $2(0.9 \%)$ had an mRS of 5. Six pa-
TABLE 5. Outcomes in 5 patients presenting with pAVMs

\begin{tabular}{lcccc}
\hline \multicolumn{1}{c}{ Outcome } & Total & $\mathrm{mRS} 0-2$ & $\mathrm{mRS} 3-6$ & $\mathrm{p}$ Value \\
\hline Recurrence & 5 & 4 & 1 & 0.48 \\
\hline $\begin{array}{l}\text { AVM obliteration on } \\
\text { follow-up imaging }\end{array}$ & $131 / 165$ & $117 / 145$ & $14 / 20$ & 0.24 \\
\hline $\begin{array}{l}\text { Seizures on } \\
\text { follow-up }\end{array}$ & 19 & 13 & 6 & 0.028 \\
\hline $\begin{array}{l}\text { Shunt on follow-up } \\
\text { Postradiation }\end{array}$ & 7 & 5 & 2 & 0.68 \\
hemorrhage & 11 & 5 & 6 & 0.002 \\
\hline $\begin{array}{l}\text { Death } \\
\text { Follow-up duration } \\
\text { (mos) }\end{array}$ & $69.7 \pm 62.1$ & $79.9 \pm 61.5$ & $78.0 \pm 67.6$ & 0.62 \\
\hline
\end{tabular}

Follow-up is expressed as the mean $\pm \mathrm{SD}$. Boldface type indicates statistical significance.

tients $(2.8 \%)$ died during follow-up. One patient died of a massive pulmonary embolism 7 months after GKRS and 1 patient died of leukemia 5 years after GKRS. One patient died of herniation due to a cerebral hemorrhage after the family declined treatment for a thalamic pAVM that had been incidentally found 2 years prior. One patient died of herniation due to hemorrhage 3 years after GKRS for a cerebellar pAVM despite an emergency craniectomy. One patient died of delayed medical complications 1 year after presenting with devastating brain hemorrhage for which he underwent a craniotomy for hematoma evacuation and resection and GKRS for residual lesion. One patient died of brainstem pAVM rupture 15 years after GKRS.

\section{Adverse Outcomes}

Eleven patients $(6 \%)$ who underwent GKRS as part of their treatment suffered from hemorrhage afterward, of whom 5 had good outcomes and 6 had poor outcomes ( $p$ $=0.002$ ). The mean first treatment GKRS dose was 20 Gy among patients without hemorrhage and 18.5 Gy among those with post-treatment hemorrhage $(\mathrm{p}=0.1)$. The mean time until postradiation hemorrhage was 2 years. Nineteen patients (9\%) had developed persistent seizure disorders by long-term follow-up, of whom 13 had good outcomes and 6 had poor outcomes $(\mathrm{p}=0.028)$. Twelve patients (5.7\%) required permanent CSF diversion, with 9 patients having a good outcome and 3 having a poor outcome $(\mathrm{p}=$ 0.39 ). Four of the patients requiring shunting had pAVMs in the basal ganglia or thalamus, 3 had lobar pAVMs, 2 had brainstem pAVMs, 2 had cerebellar pAVMs, and 1 had a pAVM of the corpus callosum. Nine had hemorrhage on presentation. The other 3 presented with obstructive hydrocephalus from mass effect (Table 5).

\section{Spetzler-Martin Grade, Treatment Type, and Long-Term Outcomes}

When stratifying by Spetzler-Martin grade, patients who had a grade of I-III and who had a craniotomy as part of their care had a mean mRS score of 1.2 on long-term follow-up, with $38(81 \%)$ of the 47 patients having a good 


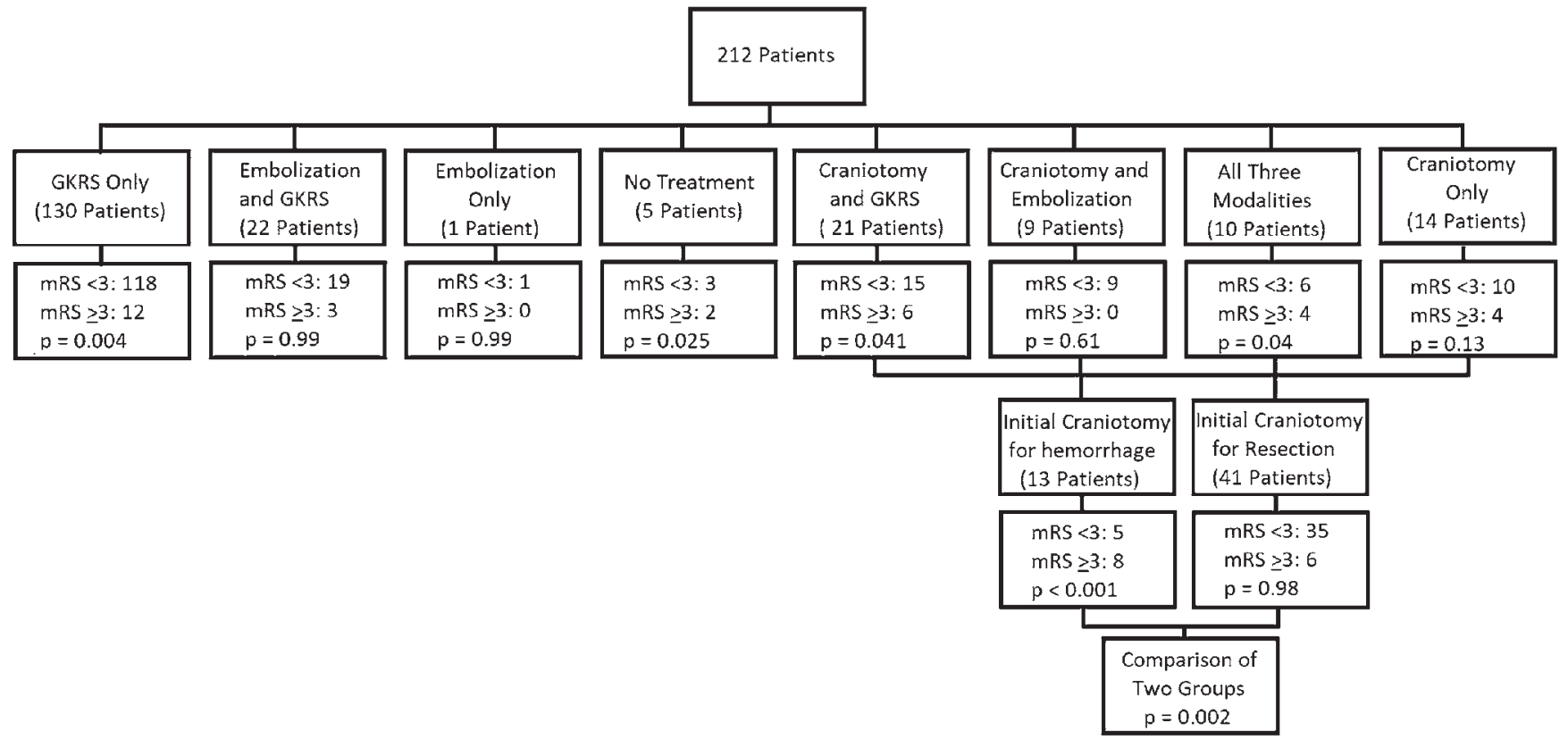

FIG. 1. Comparison of good ( $m R S<3$ ) and poor ( $m R S \geq 3$ ) outcomes by treatment type. The $p$ values represent comparison of the designated group to the remainder of the cohort except where otherwise indicated.

outcome. In contrast, patients who underwent GKRS alone with a grade I-III pAVM had a mean mRS score of 0.7 on long-term follow-up, with 83 (93\%) of 89 patients having a good outcome $(\mathrm{p}=0.028)$. However, when considering patients who underwent resection without a hemorrhagic presentation, the mean mRS score on follow-up was 0.55 versus a mean of 1.4 among those with a hemorrhagic presentation $(\mathrm{p}=0.002)$. Patients with a Spetzler-Martin grade IV-V pAVM who underwent a craniotomy as part of their care had a mean mRS score of 3.7, with $2(29 \%)$ of 7 patients having a good outcome. In contrast, patients who underwent GKRS alone with a grade IV-V pAVM had a mean mRS of 1.1 on long-term follow-up, with 35 $(85 \%)$ of 41 patients having a good outcome $(p=0.0044)$. All of the patients who underwent craniotomy with a highgrade AVM presented with hemorrhage requiring evacuation. Thirteen patients who underwent craniotomy initially for decompression of hemorrhage subsequently had other treatment. When excluding these patients, the mean long-term mRS score for the remaining patients with craniotomy was 1.1 (Spetzler-Martin grade I-III: mean mRS 1, Spetzler-Martin grade IV-V: mean mRS 2) versus 2.8 (Spetzler-Martin grade I-III: mean mRS 1.9, SpetzlerMartin grade IV-V: mean mRS 4.6) for the 13 excluded patients $(\mathrm{p}=0.002)$ (Fig. 1). Excluding these 13 patients, there was no difference in outcomes between craniotomy and GKRS for any Spetzler-Martin grade $(\mathrm{p}=0.37)$.

\section{Multivariate Analysis}

Independent variables with an association $(p<0.2)$ with outcome on univariate analysis were assessed via multivariate logistic regression. The variables included in the multivariate model were age, presenting mRS score, hemorrhagic presentation, number of draining veins, lack of pAVM treatment, craniotomy as part of treatment for hemorrhage, Spetzler-Martin grade, and post-GKRS hemorrhage. Hemorrhagic presentation, vein number, and the presence of a craniotomy were not found to be independent predictors of outcome. Increasing age was found to trend toward an association with a decreased probability of poor outcome (OR $0.9, \mathrm{p}=0.08)$. No pAVM treatment (OR 18.9, $\mathrm{p}=0.02$ ), need for a craniotomy for hemorrhagic decompression (OR $6, \mathrm{p}=0.03$ ), post-treatment hemorrhage (OR 4.3, $\mathrm{p}=0.12$ ), post-treatment seizure disorder (OR 2.9, $\mathrm{p}=0.15$ ), higher preoperative mRS score (OR 2.1, $\mathrm{p}=0.0004)$, and higher Spetzler-Martin score (OR 1.8, $\mathrm{p}$ $=0.0005$ ) were associated with or trended toward association with poor outcome (Table 6).

\section{Discussion}

In this report we demonstrate the following: 1) the multimodality management of pediatric pAVMs is associated with a high rate of long-term functional independence and obliteration; 2) recurrences, while rare, frequently occur many years after obliteration; and 3) the strategic application of different treatment modalities is not independently associated with a better or worse outcome when applied to individual patient circumstances such as hemorrhagic presentation or high Spetzler-Martin grade lesions.

Eighty-five percent of our cohort were functionally independent on long-term follow-up, with a total of 6 deaths, which reflects a superior outcome compared to older cohorts but is comparable to more recent ones. ${ }^{1,11,12}$ Of the deaths, 3 were directly related to brain hemorrhage from pAVMs, 2 were associated with unrelated medical con- 
TABLE 6. Multivariate analysis assessing predictors of poor outcome (follow-up mRS score $>2$ )

\begin{tabular}{lccl}
\hline \multicolumn{1}{c}{ Variable } & OR & $\mathrm{Cl}$ & $\mathrm{p}$ Value \\
\hline Age & 0.9 & $0.8-1.01$ & 0.08 \\
\hline Preop mRS score & 2.1 & $1.4-3.2$ & $\mathbf{0 . 0 0 0 4}$ \\
\hline Spetzler-Martin grade & 1.8 & $1.3-2.4$ & $\mathbf{0 . 0 0 0 5}$ \\
\hline Vein no. & 1.3 & $0.59-3.0$ & 0.5 \\
\hline No AVM treatment & 18.9 & $1.6-218.7$ & $\mathbf{0 . 0 2}$ \\
\hline Hemorrhagic presentation & 0.96 & $0.22-4.3$ & 0.96 \\
\hline Craniotomy for hemorrhage & 6 & $1.2-29.4$ & $\mathbf{0 . 0 3}$ \\
\hline Post-treatment hemorrhage & 4.3 & $0.7-26.6$ & 0.12 \\
\hline Postop seizure disorder & 2.9 & $0.69-11.8$ & 0.15 \\
\hline
\end{tabular}

Boldface type indicates statistical significance.

ditions that developed after pAVM treatment, and 1 was due to a hematological malignancy. Overall, no differences in outcome based on treatment modality were identified. However, this was in part due to selective application and combination of techniques based on individual cases. GKRS was found to be effective in the management of many low and high Spetzler-Martin grade lesions, with craniotomies being predominantly reserved for low-grade lesions and emergency decompression. As such, patients with high-grade lesions are routinely advised to pursue nonresective treatment, whereas situations in which the patient is unstable due to hemorrhagic presentation are routinely managed with craniotomy. Families of patients with low-grade lesions are offered both craniotomy and GKRS options, with deference to personal preference in situations of equipoise. High-density niduses are believed to be particularly ideal for GKRS. Embolization has gradually become seen as a secondary option in situations in which deep arterial feeders can be accessed in order to reduce operative complexity and in situations in which high-risk features such as arterial aneurysms are noted. Overall, however, patients are provided the opportunity to go forward in life to achieve their potential as we hope will happen for all of our patients (Fig. 2).

In our study, approximately $80 \%$ of patients with radiographic follow-up demonstrated obliteration. Most obliteration rates in the pAVM literature hover around $60 \%-$ $70 \%{ }^{12-14}$ However, substantial differences in the definition of obliteration and the accepted radiographic modalities exist. The majority of our patients had a documented formal angiogram after treatment. It is our general practice to rely on MRA for post-treatment screening purposes in order to minimize the radiation burden, but a formal angiogram after treatment is recommended. Typically, we follow patients who undergo GKRS for 3 years with MRA. At this point, if the AVM is no longer noted on MRA, a formal angiogram is used to confirm this. If residual pAVM is noted, an angiogram may be performed as part of additional treatments. For patients who underwent other treatment modalities, an angiogram 6-12 months after treatment is recommended. For all patients, MRA annually is typically recommended, although for patients

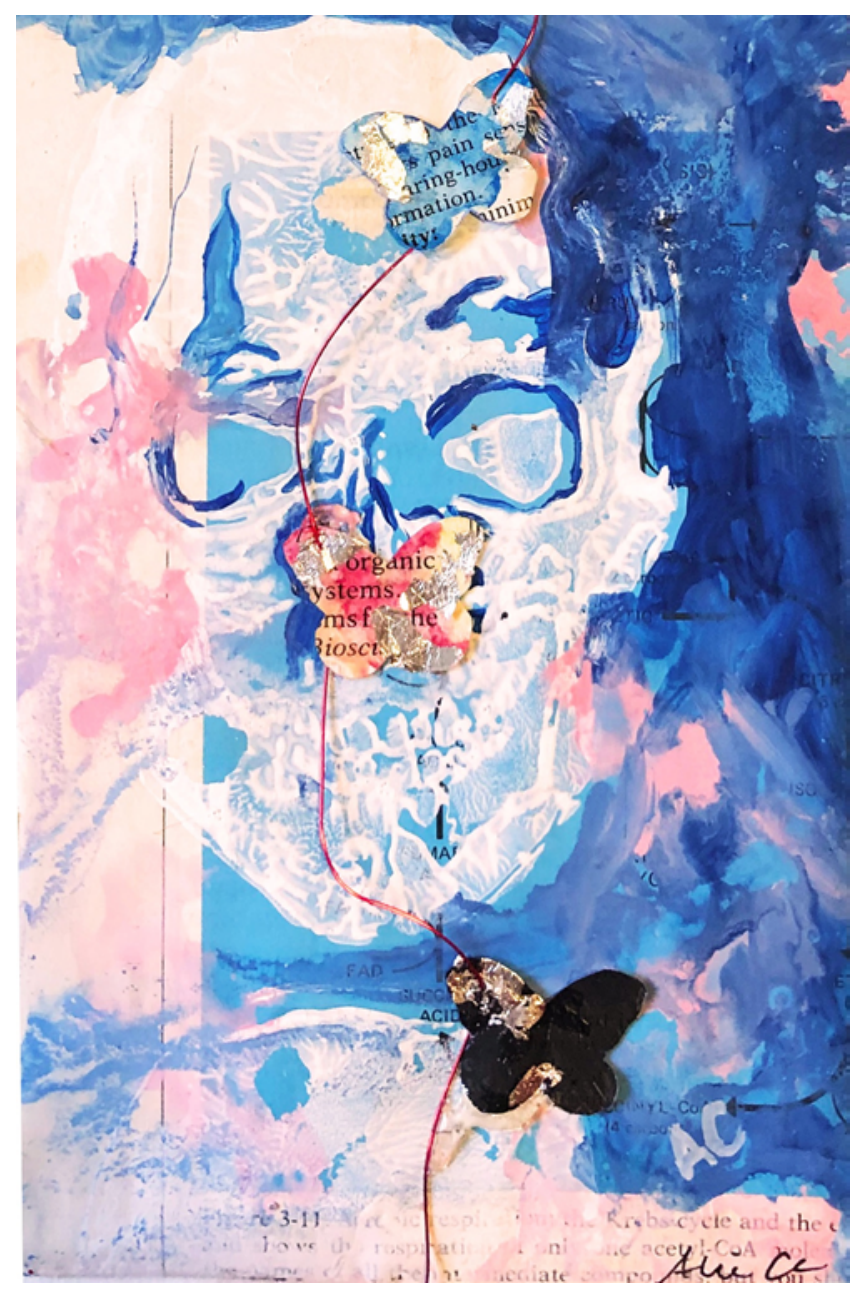

FIG. 2. Artistic rendition of pAVMs by a former patient presenting with hemorrhagic PAVM, treated by our center, who went on to become a professional graphic designer and artist in adulthood. Copyright Michael M. McDowell and Stephanie Greene. Published with permission. Figure is available in color online only.

with exceptionally long follow-up this may be gradually spaced out to every 3 years. Of note, the mean dose for GKRS was $20 \mathrm{~Gy}$ in this series. In a recent study, Starke et al. demonstrated that marginal GKRS doses of 22 Gy or higher appeared to be associated with higher rates of obliteration. ${ }^{8}$ In-field obliteration has been correlated to marginal dosing, but this may in part be secondary to higher doses reducing the volumetric inaccuracy of AVM nidus localization during angiogram-based radiosurgery. ${ }^{15,16}$

Recurrence separate from residual pAVM was noted in 5 cases during follow-up. Recurrence was frequently found many years after documented obliteration and may potentially increase in frequency during the lifespan of this cohort. We recommend continued imaging follow-up beyond obliteration every 3-5 years in pediatric patients. In most cases, MRA is the most appropriate modality for screening, with formal angiography reserved for concerning findings. Recurrence was found to be associated with craniotomies without GKRS in the context of hemorrhagic 
presentation. Microsurgery may not allow for the visualization of all aspects of abnormal microvasculature during resection in a population with potentially higher capacity for vascular neogenesis and thus regrowth of pAVMs. Hemorrhage may reflect a higher concentration of local growth factors, prompting the development of friable vessels prone to bleeding and also resulting in recurrence despite complete resection.

Our overall hemorrhage rate was consistent with past literature. Hemorrhage was not found to be independently associated with poor outcome. ${ }^{1,3,4,11,17,18}$ This should come with the caveat that multivariate analysis does not provide insight into causality, merely the strength of association. Of patients presenting without hemorrhage, only 3 of 84 presented with an mRS score $>2$, versus 62 of the 128 patients presenting with hemorrhage $(\mathrm{p}<0.0001)$. However, although hemorrhage was strongly correlated with a higher mRS score, a hemorrhagic presentation without an initially elevated mRS severe disability was unlikely to result in a poor outcome. Hemorrhage also was associated on univariate analysis with poor outcomes in children under the age of 10 years, in part due to 4 of the 6 deaths occurring in younger children. This group also had a higher proportion of hemorrhagic presentation in comparison to older patients, potentially in part due to a lower tendency to notice subtle neurological signs (such as headaches) in very young children. In addition, unstable AVMs may be more likely to present at an early age due to a greater bleeding tendency. Ultimately, patients with hemorrhage frequently benefit from craniotomy for both decompressive and therapeutic reasons. However, these patients may also be particularly vulnerable to having residual or recurrent pAVMs, in which case GKRS should be considered as an alternative to repeat craniotomy. Craniotomies instead of GKRS for definitive resection of low Spetzler-Martin grade, unruptured lesions remain a viable option and should be presented to families during discussion of treatment options, particularly in patients with angiographic features concerning for hemorrhage risk, such as aneurysms.

On multivariate analysis, post-GKRS hemorrhage trended toward an association with poor outcome. PostGKRS hemorrhage is relatively uncommon, but has been documented in a large multicenter database in up to $9 \%$ of pediatric cases. ${ }^{17}$ In our cohort, hemorrhage occurred in $5.5 \%$ of patients who underwent GKRS, with the mean time until hemorrhage being 2 years after initial treatment. Post-GKRS hemorrhage warrants counseling in cases in which therapeutic equipoise may exist. Nonetheless, our data demonstrate that GKRS overall is a valuable and safe treatment option, particularly in high-grade lesions in which surgical and endovascular management are not tenable.

\section{Conclusions}

High rates of long-term functional independence can be achieved with comprehensive multimodality treatment of pAVMs. At our center, we did not find a difference in outcome based on treatment choice when accounting for factors such as Spetzler-Martin grade and presenting morbidity from hemorrhage. Recurrences, although infrequent, typically occur years after obliteration, and thus "cured" patients warrant long-term screening.

\section{Acknowledgments}

We thank Alessandra Crivelli, a pAVM survivor, for her artwork contribution.

\section{References}

1. Anderson RC, McDowell MM, Kellner CP, et al. Arteriovenous malformation-associated aneurysms in the pediatric population. J Neurosurg Pediatr. 2012;9(1):11-16.

2. Hoffman C, Riina HA, Stieg P, et al. Associated aneurysms in pediatric arteriovenous malformations and the implications for treatment. Neurosurgery. 2011;69(2):315-322.

3. Hofmeister C, Stapf C, Hartmann A, et al. Demographic, morphological, and clinical characteristics of 1289 patients with brain arteriovenous malformation. Stroke. 2000;31(6):1307-1310.

4. Kellner CP, McDowell MM, Phan MQ, et al. Number and location of draining veins in pediatric arteriovenous malformations: association with hemorrhage. J Neurosurg Pediatr. 2014;14(5):538-545.

5. Klimo P Jr, Rao G, Brockmeyer D. Pediatric arteriovenous malformations: a 15-year experience with an emphasis on residual and recurrent lesions. Childs Nerv Syst. 2007;23(1):3137.

6. Maher CO, Scott RM. Linear vein-based arteriovenous malformations in children. $J$ Neurosurg Pediatr. 2009;4(1):12-16.

7. Soltanolkotabi M, Schoeneman SE, Alden TD, et al. Onyx embolization of intracranial arteriovenous malformations in pediatric patients. J Neurosurg Pediatr. 2013;11(4):431-437.

8. Starke RM, Ding D, Kano H, et al. International multicenter cohort study of pediatric brain arteriovenous malformations. Part 2: Outcomes after stereotactic radiosurgery. J Neurosurg Pediatr. 2017;19(2):136-148.

9. Kondziolka D, Lunsford LD, Flickinger JC. Stereotactic radiosurgery in children and adolescents. Pediatr Neurosurg. 1990-1991;16(4-5):219-221.

10. Zhao H, Collier JM, Quah DM, et al. The modified Rankin Scale in acute stroke has good inter-rater-reliability but questionable validity. Cerebrovasc Dis. 2010;29(2):188-193.

11. Oulasvirta E, Koroknay-Pál P, Hafez A, et al. Characteristics and long-term outcome of 127 children with cerebral arteriovenous malformations. Neurosurgery. 2019;84(1):151-159.

12. Yang W, Anderson-Keightly H, Westbroek EM, et al. Longterm hemorrhagic risk in pediatric patients with arteriovenous malformations. J Neurosurg Pediatr. 2016;18(3):329_ 338.

13. Morgenstern PF, Hoffman CE, Kocharian G, et al. Postoperative imaging for detection of recurrent arteriovenous malformations in children. J Neurosurg Pediatr. 2016;17(2):134140.

14. Umansky D, Corn BW, Strauss I, et al. Combined treatment approach to cerebral arteriovenous malformation in pediatric patients: stereotactic radiosurgery to partially Onyx-embolized AVM. Childs Nerv Syst. 2018;34(11):2269-2274.

15. Flickinger JC, Kondziolka D, Maitz AH, Lunsford LD. An analysis of the dose-response for arteriovenous malformation radiosurgery and other factors affecting obliteration. Radiother Oncol. 2002;63(3):347-354.

16. Flickinger JC, Pollock BE, Kondziolka D, Lunsford LD. A dose-response analysis of arteriovenous malformation obliteration after radiosurgery. Int J Radiat Oncol Biol Phys. 1996;36(4):873-879.

17. Chen CJ, Ding D, Kano H, et al. Stereotactic radiosurgery for pediatric versus adult brain arteriovenous malformations. Stroke. 2018;49(8):1939-1945. 
18. Fullerton HJ, Achrol AS, Johnston SC, et al. Long-term hemorrhage risk in children versus adults with brain arteriovenous malformations. Stroke. 2005;36(10):2099-2104.

\section{Disclosures}

Dr. Agarwal receives royalties from Thieme Medical Publishers. Dr. Lunsford is a consultant for Insightec and DSMB, and he has direct stock ownership in Elekta.

\section{Author Contributions}

Conception and design: McDowell, Agarwal, Greene. Acquisition of data: McDowell, Agarwal, Mao, Johnson, Kano, Greene. Analysis and interpretation of data: McDowell, Agarwal, Mao,
Johnson, Kano, Greene. Drafting the article: all authors. Critically revising the article: all authors. Reviewed submitted version of manuscript: all authors. Approved the final version of the manuscript on behalf of all authors: McDowell. Statistical analysis: McDowell.

\section{Correspondence}

Michael M. McDowell: University of Pittsburgh Medical Center, Pittsburgh,PA.mcdowellmm2@upmc.edu. 